\title{
X-ray emission in the direction of the SNR G318.2+0.1
}

\author{
F. Bocchino ${ }^{1,2}$, A. N. Parmar ${ }^{1}$, S. Mereghetti ${ }^{3}$, M. Orlandini $^{4}$, A. Santangelo ${ }^{5}$, and L. Angelini ${ }^{6}$ \\ 1 Astrophysics Division, Space Science Dept. of ESA, ESTEC, Postbus 299, 2200 AG Noordwijk, The Netherlands \\ 2 Osservatorio Astronomico di Palermo, Piazza del Parlamento 1, 90134 Palermo, Italy \\ 3 Istituto di Fisica Cosmica e Tecnologie Relative, CNR, Via Bassini 15, 20133 Milano, Italy \\ ${ }^{4}$ Istituto Tecnologie e Studio Radiazioni Extraterrestri, CNR, Via Gobetti 101, 40129 Bologna, Italy \\ 5 Istituto di Fisica Cosmica ed Applicazioni all'Informatica, CNR, Via U. La Malfa 153, 90146 Palermo, Italy \\ ${ }^{6}$ Laboratory for High Energy Astrophysics, Code 660.2, NASA/Goddard Space Flight Center, MD 20771, USA
}

Received 3 July 2000 / Accepted 24 November 2000

\begin{abstract}
We report the discovery of three X-ray sources within the radio shell G318.2+0.1, one of which may be extended. Two of the sources were detected during the BeppoSAX Galactic Plane Survey and one was found in archival ROSAT data. The fainter BeppoSAX source is coincident with an ultra-compact galactic $\mathrm{H}$ II region, and we discuss the possibility that it can be a flaring young stellar object, while the other BeppoSAX source has no obvious counterpart. The PSPC source is consistent with emission from a foreground star. The hard spectrum of the brighter BeppoSAX source is consistent with a non-thermal origin, although a thermal nature cannot be formally excluded. If this source is associated with G318.2+0.1, then its hard spectrum suggests that it may be site of non-thermal electron acceleration.
\end{abstract}

Key words. supernovae: general; ISM: individual object: G318.2+0.1; ISM: supernova remnants; X-rays: ISM

\section{Introduction}

G318.2+0.1 is a large $\left(40^{\prime} \times 35^{\prime}\right)$ radio shell discovered by Whiteoak \& Green (1996) in the MOST $843 \mathrm{MHz}$ survey. The remnant was identified by means of the criterium of the ratio of $60 \mu \mathrm{m}$ to radio flux density, but Whiteoak \& Green (1996) have also reported of additional polarization measurement which seems to confirm its nature. This object is mainly composed of two non-thermal filaments at the North-West and South-East which form two sections of the shell, plus a central region and a bright clump to the South-West, which are thermal and correspond to two H II regions. Moreover, the ultra-compact (UC) H II region IRAS14498-5856, reported in Walsh et al. (1997), is also located towards the radio shell; this UC H II region is a methanol, $\mathrm{OH}$ and $\mathrm{H}_{2} \mathrm{O}$ maser (GAL318.05+0.08, Gaylard \& MacLeod 1993). As many other newly discovered radio shells, very little is known about G318.2+0.1. In particular, we do not know its age and its distance, and the relation between the SNR and $\mathrm{H}$ II region detected in radio is still to be studied.

The X-ray band offers a unique opportunity to confirm the detection of newly discovered radio SNRs. In fact, SNRs may be strong X-ray emitters since their initial

Send offprint requests to: F. Bocchino,

e-mail: fbocchin@estec.esa.nl evolutionary stage, and several SNRs have been discovered, or rediscovered, in large area surveys or in pointed observations by X-ray satellites (see e.g., Aschenbach et al. 1991; Pfeffermann \& Aschenbach 1996). The detection of radio SNRs in other bands is important in order to properly assess the nature of these objects, as well as to provide a secure identification in cases where this is uncertain. Among other things, X-ray studies may provide information about the distance, which is a key parameter for SNRs, measured with difficulty in the radio band.

In this paper we report the discovery of three X-ray sources within the radio shell G318.2+0.1. Two are sources discovered during the BeppoSAX Galactic Plane Survey (GPS), one of which is positional coincident with the UC $\mathrm{H}$ II region, and one was found in archival ROSAT data. In Sect. 2 we describe the observations, in Sect. 3 we introduce the detected sources and in Sect. 4 we discuss their possible nature and relation to G318.2+0.1.

\section{Observations}

The BeppoSAX GPS consisted of 14 pointed observations of a region of the galactic plane between approximately $l=310^{\circ}$ to $l=320^{\circ}$ within about $\pm 1^{\circ}$ from the plane. Due to a lack of suitable guide stars the region between $l=312^{\circ}$ to $l=316^{\circ}$ could not be observed. 
The prime instruments for the survey were the MediumEnergy Concentrator Spectrometer (MECS; 1.8-10 keV; Boella et al. 1997) and the Low-Energy Concentrator Spectrometer (LECS; 0.1-10 keV; Parmar et al. 1997). The MECS consists of two grazing incidence telescopes with imaging gas scintillation proportional counters in their focal planes. The LECS uses an identical concentrator system as the MECS, but utilizes an ultra-thin entrance window and a driftless configuration to extend the low-energy response to $0.1 \mathrm{keV}$. The fields of view (FOV) of the LECS and MECS are circular with diameters of $37^{\prime}$ and $56^{\prime}$, respectively. In the overlapping energy range, the position resolution of both instruments is similar and corresponds to $90 \%$ encircled energy within a radius of $2 ! 5$ at $1.5 \mathrm{keV}$. In addition, the BeppoSAX payload includes two high energy instruments - the High Pressure Gas Scintillation Proportional Counter (HPGSPC; 5-120 keV; Manzo et al. 1997) and the Phoswich Detection System (PDS; 15-300 keV; Frontera et al. 1997). The non-imaging HPGSPC consists of a single unit with a collimator that remained on-source during the GPS observations. The non-imaging PDS consists of four independent units arranged in pairs each having a separate collimator. Each collimator was alternatively rocked on- and $210^{\prime}$ off-source every $96 \mathrm{~s}$ during the observations.

The region of sky containing G318.2+0.1 was observed between 1999 July 21 16:58 and July 22 06:56 UTC. The (J2000) pointing direction was $\mathrm{RA}=14^{\mathrm{h}} 53^{\mathrm{m}} 41.7$, Dec $=-58^{\circ} 59^{\prime} 24^{\prime \prime}$. In order to avoid solar scattered emission and other contaminating effects, data were screened retaining intervals when the elevation angle above the Earth's limb was $>4^{\circ}$ and when the instrument configurations were nominal. The screened exposures in the LECS and MECS are $10.9 \mathrm{ks}$ and $25.9 \mathrm{ks}$, respectively. The MECS $0.5-10 \mathrm{keV}$ image is shown in Fig. 1. The image was smoothed using a Gaussian filter with a $\sigma$ of 1.'5.

Inspection of PDS data after having subtracted a contaminating source present in one of the offset collimator positions does not reveal the presence of any excess signal above background. The $3 \sigma$ confidence upper limit to any $15-30 \mathrm{keV}$ count rate is $<0.17 \mathrm{cts} \mathrm{s}^{-1}$. For a Crablike spectrum this corresponds to $<2 \mathrm{mCrab}$. As for the HPGSPC, we have derived a $3 \sigma$ confidence upper limit of $0.25 \mathrm{~s}^{-1}$ in the $10-20 \mathrm{keV}$ band, which correspond to $3.5 \mathrm{mCrab}$.

In addition, we have used two ROSAT Position Sensitive Proportional Counter (PSPC) archival observations (RP500096 and RP500192 with exposures of 1.3 and $4.5 \mathrm{ks}$, respectively) both pointed at $\mathrm{RA}=14^{\mathrm{h}} 58^{\mathrm{m}} 19.2$, Dec $=-58^{\circ} 27^{\prime} 00^{\prime \prime}(\mathrm{J} 2000)$ which partially overlaps the radio shell of G318.2+0.1. The PSPC, which covers the $0.1-2.4 \mathrm{keV}$ energy range, has a circular FOV of $\sim 2^{\circ}$ and a spatial resolution of $\sim 30^{\prime \prime}$ full width half-maximum (FWHM) on-axis and $\sim 2$ '.5 at $45^{\prime}$ off-axis, near the location of one of the source discussed below. The PSPC spectral resolution is $E / \Delta E \sim 2$ at $1 \mathrm{keV}$. The FOVs of the BeppoSAX and ROSAT observations used here do not overlap.

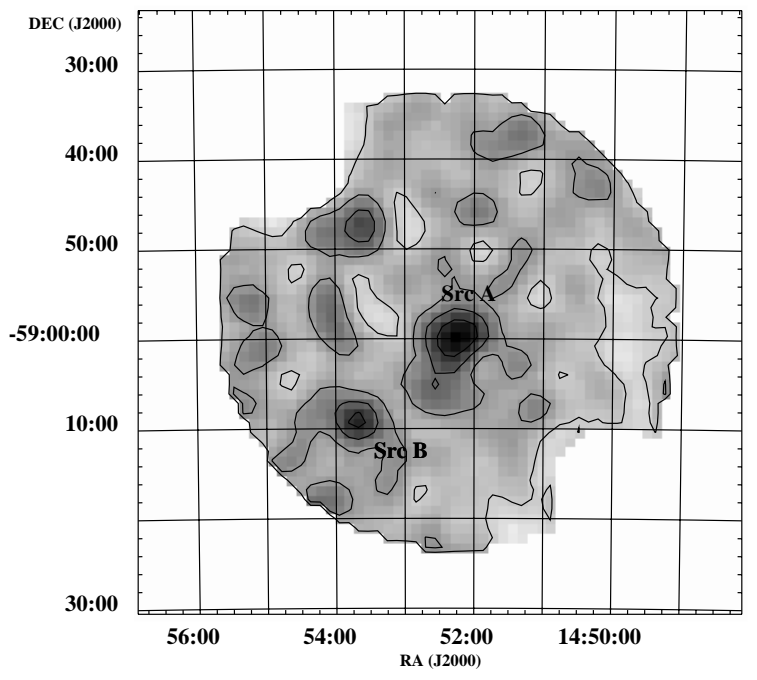

Fig. 1. Background subtracted and vignetting corrected 2-10 keV MECS image of the BeppoSAX GPS observation which included part of the radio shell G318.2+0.1. Contour levels represent $20 \%, 40 \%, 60 \%$, and $80 \%$ of the peak intensity of $0.21 \mathrm{cts} \mathrm{ks}^{-1} \operatorname{arcmin}^{-2}$

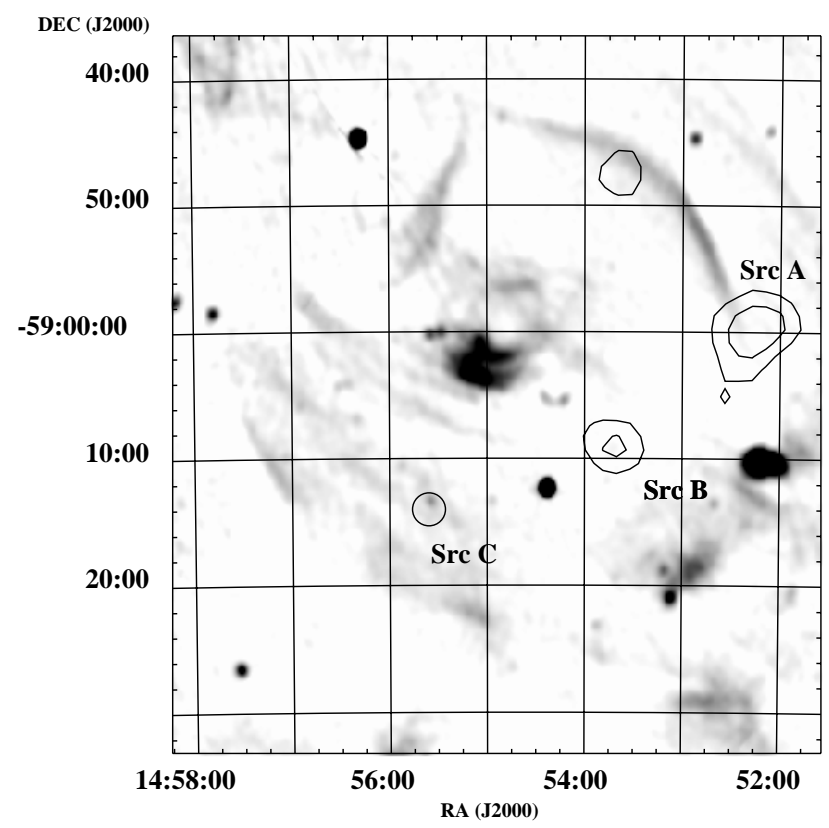

Fig. 2. $843 \mathrm{MHz}$ MOST survey image of the radio shell G318.2+0.1 (from Whiteoak \& Green 1996). The 60\%, and $80 \%$ of the peak contour levels of the BeppoSAX image which mark the locations of sources A and B are overlaid. A 1'? radius circle marks the position of PSPC source $\mathrm{C}$ with its uncertainty

\section{X-ray sources inside $\mathrm{G} 318.2+0.1$}

\subsection{Source detection}

Inside the radio boundary of G318.2+0.1, we have detected the three X-ray sources which are listed in Table 1 together with their positions, count rates and significances. In Fig. 2, we reproduce the Whiteoak \& Green (1996) radio map of G318.2+0.1 with the position of the X-ray sources superimposed. Sources A and B were 
Table 1. List of X-ray sources towards G318.2+0.1. The count rate is given in the energy range $2-10 \mathrm{keV}$ (MECS) or $0.2-2.0 \mathrm{keV}$ (PSPC). Vignetting correction and background subtraction have been applied. Position uncertainty is given as the width of $90 \%$ confidence range

\begin{tabular}{lcccccc}
\hline Source & Inst & RA & Dec & $\begin{array}{c}\text { Uncertainty } \\
\text { radius }\left(^{\prime}\right)\end{array}$ & $\begin{array}{c}\text { Count rate } \\
\left(\mathrm{s}^{-1}\right)\end{array}$ & Significance \\
\hline A & MECS & $14^{\mathrm{h}} 52^{\mathrm{m}} 12.4^{\mathrm{s}}$ & $-58^{\mathrm{d}} 59^{\prime} 40^{\prime \prime}$ & 1 & $(1.05 \pm 0.11) 10^{-2}$ & $6 \sigma$ \\
$\mathrm{B}$ & MECS & $14^{\mathrm{h}} 53^{\mathrm{m}} 39.5^{\mathrm{s}}$ & $-59^{\mathrm{d}} 08^{\prime} 52^{\prime \prime}$ & 1 & $(8.2 \pm 1.6) 10^{-3}$ & $3 \sigma$ \\
$\mathrm{C}$ & PSPC & $14^{\mathrm{h}} 55^{\mathrm{m}} 35.5^{\mathrm{s}}$ & $-59^{\mathrm{d}} 12^{\prime} 42^{\prime \prime}$ & 1.3 & $0.115 \pm 0.008$ & $27 \sigma$ \\
\hline
\end{tabular}

detected using the sliding-cell method of the XIMAGE software package (Giommi et al. 1992) on the $32^{\prime \prime}$ pixel BeppoSAX MECS image. Some fainter X-ray emission is also present in the North-Western part of the radio shell but it is not detected above the $3 \sigma$ level and we will not discuss it further. Source A is the brightest and is located at the rim of the shell, between the North-West shell filament and the bright thermal radio clump at $14^{\mathrm{h}} 52^{\mathrm{m}} 09^{\mathrm{s}}$, $-59^{\circ} 10^{\prime}$ discussed in Whiteoak \& Green (1996). The $80 \%$ (i.e., the innermost), $60 \%$ and $40 \%$ contour levels around Source A show some evidence of source elongation from the North-West to the South-East which is not shown in the corresponding image of $\mathrm{Cyg} \mathrm{X}-1$, a bright point source used as a template for the instrumental Point Spread Function (PSF). We therefore suggest that Source A may be extended. Source B is positioned roughly halfway between the shell and the core, with no obvious radio counterpart at $843 \mathrm{MHz}$, and it is consistent with being pointlike. No sources were detected in the LECS image, with a $3 \sigma$ upper limit of $2.710^{-3}$ cts s $^{-1}$ in a $8^{\prime}$ radius circle. This may be due to the much lower LECS exposure time and/or to high X-ray absorption, which supress X-rays below $2 \mathrm{keV}$, the only energy band where the LECS is more sensitive than the MECS.

Source C was detected in the ROSAT All-Sky Survey (RASS, 1RXS J145540.4-591320, Voges et al. 1999) and it is also present in the WGACAT (1WGA J1455.5-5912 and 1WGA J1455.7-5912, White et al. 1994). The count rates measured in different $R O S A T$ observations are $0.11 \pm$ $0.02,0.11 \pm 0.01$ and $0.115 \pm 0.008$ cts s $^{-1}$ in the RASS (October 1990), RP500192 (March 1992) and RP500096 (February 1993), respectively. The source has a shape severely distorted by mirror blurring since it is located at $\sim 50^{\prime}$ off-axis, but its extension is compatible with the size of the PSPC PSF at that off-axis angle.

In order to investigate the nature of the detected Xray source (and therefore the relation with G318.2+0.1), we have performed source cross-identifications with catalogues using SIMBAD and a search radius of $5^{\prime}$. Sources $\mathrm{A}$ and $\mathrm{B}$ are coincident with very weak IRAS sources at $12 \mu \mathrm{m}$. However, this band is heavily confused by the galactic background. Source B is the only one which has a strong counterpart at 25, 60 and $100 \mu \mathrm{m}$ and is also coincident with the UC H II region IRAS 14498-5856 reported by Walsh et al. (1997). As for Source C, the star GSC 08693-00036, with $B=11.4$ and $V=10.7$ falls inside its error circle, at $1^{\prime}$ offset. We also inspected the
Digital Sky Survey image within $2^{\prime}$ from the positions of the sources and we did not find any other obvious counterparts (except those already found with SIMBAD).

\subsection{Spectral analysis}

Since the sources are weak, the analysis of their hardness ratios is a very good method to constrain the X-ray emission process. For the MECS sources, we defined two hardness ratios in the following way:

$$
H R 1=\frac{H_{1}-S_{1}}{H_{1}+S_{1}} ; \quad H R 2=\frac{H_{2}-S_{2}}{H_{2}+S_{2}}
$$

where $S_{1}$ is the number of counts in the $1.0-2.0 \mathrm{keV}$ band, $H_{1}$ in the $2.0-10 \mathrm{keV}$ band, $S_{2}$ in the $2.0-5.0 \mathrm{keV}$ band and $H_{2} 5.0-10 \mathrm{keV}$ band. The counts are collected in circular area with $4^{\prime}$ and $2^{\prime}$ radius for Source A and B, respectively, and they were background subtracted using the MECS standard background maps, because we have verified that the background collected in the short exposure observations of G318.2+0.1 is statistically poor. Errors in the $H$ and $S$ quantities are computed propagating the Poissonian errors. The uncertainties on the ratios are computed using the maximum and minimum values that the denominators and the numerators can assume considering their respective uncertainties. The values of $H R 1$ and HR2 for Source A and Source B and their uncertainties are graphically represented in Fig. 3. In the same figure, we have also plotted the expected $H R 1$ and $H R 2$ values for a power-law and an optically thin thermal plasma (MEKAL) emission model with many different values of the powerlaw photon index $(\gamma)$, the plasma temperature $(T)$ and the intervening absorbing column $\left(N_{\mathrm{H}}\right)$, computed with simulations using XSPEC.

Source A is located in a region of the $H R 1$ and $H R 2$ plane which correspond to very hard spectra. In particular, the non-thermal power law seems to be preferred over the thermal plasma model, since for Source A to be of thermal origin its temperature should be $>10^{8}-10^{9} \mathrm{~K}$, which seems unreasonable high. The $N_{\mathrm{H}}$ of Source A is poorly constrained by this analysis, since we have only an upper limit between $10^{22}$ and $10^{23} \mathrm{~cm}^{-2}$. We recall however, that MECS is not the best suited instrument to measure low values of $N_{\mathrm{H}}$ accurately, because its effective area drops quickly to zero below $1 \mathrm{keV}$. This is shown in Fig. 3 by the proximity of the symbols corresponding to $10^{20}$ and $10^{21} \mathrm{~cm}^{-2}$. As for Source B, the associated 


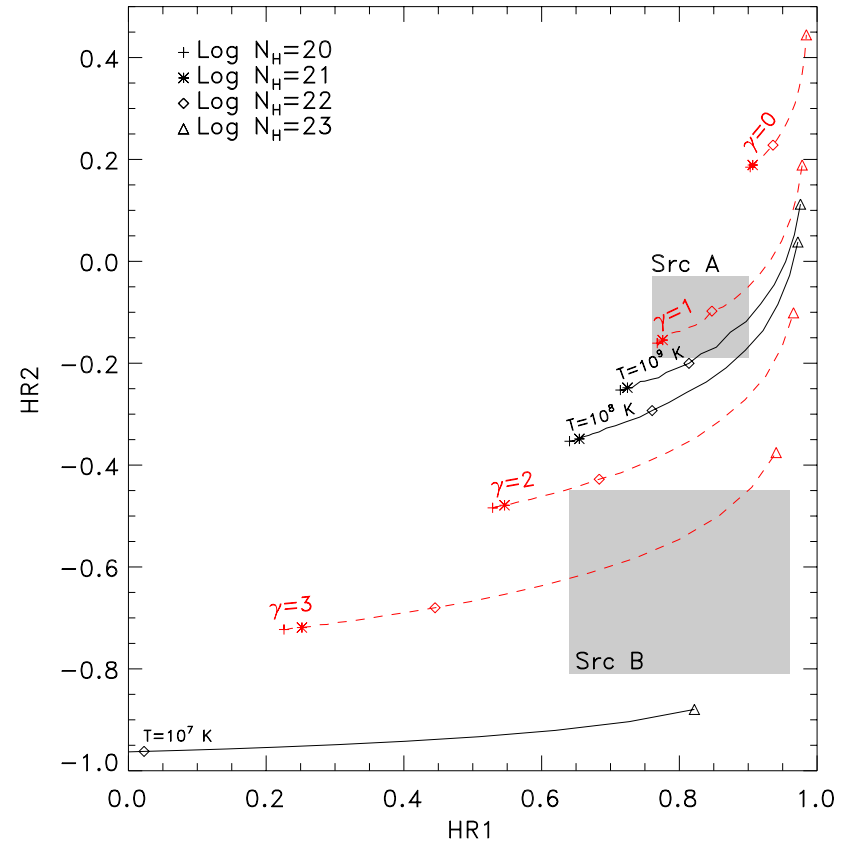

Fig. 3. Observed Source A and Source B MECS hardness ratios with uncertainties (shaded areas) and values expected from a power-law (gray and dashed lines) and MEKAL (solid lines) emission model with different parameters values (derived by simulations). The lines are constant temperature or power-law index loci, while symbols represent $N_{\mathrm{H}}$ values

hardness ratios show that it is significantly softer than Source A. It could be a non-thermal source with $\gamma \sim 3$, or a thermal source with a temperature of a few $10^{7} \mathrm{~K}$. In any case, it seems to be very absorbed, with $N_{\mathrm{H}}>10^{22} \mathrm{~cm}^{-2}$.

We have also attempted to extract the spectrum of Source A, using the same extraction region and background used for hardness ratios. We have used the LECS even in absence of Source A detection in order to provide a more stringent lower limit to the value of interstellar absorption. In fact, as the hardness ratio scatter plot suggests, MECS data yields only an upper limit to the $N_{\mathrm{H}}$, while the inclusion of LECS yields also a lower limit to this parameter. The spectra were rebinned to oversample the FWHM of the energy resolution by a factor 3 and to have additionally a minimum of 30 counts per bin to allow use of the $\chi^{2}$ statistic. Data were selected in the energy range $0.1-4 \mathrm{keV}$ for the LECS and 1.8-10 keV for the MECS. The number of LECS and MECS counts in the spectra after background subtraction is 20 and 307. The photo-electric absorption cross sections of Morrison \& McCammon (1983) and the solar abundances of Anders \& Grevesse (1989) are used throughout.

Absorbed optically thin thermal plasma (MEKAL in XSPEC), power-law, thermal bremsstrahlung and blackbody models were tried. At first, we used a free normalization constant between LECS and MECS. Since the fitted values of the constant were between 0.7 and 2.0, we fixed its value to 1 . All models provide an adequate

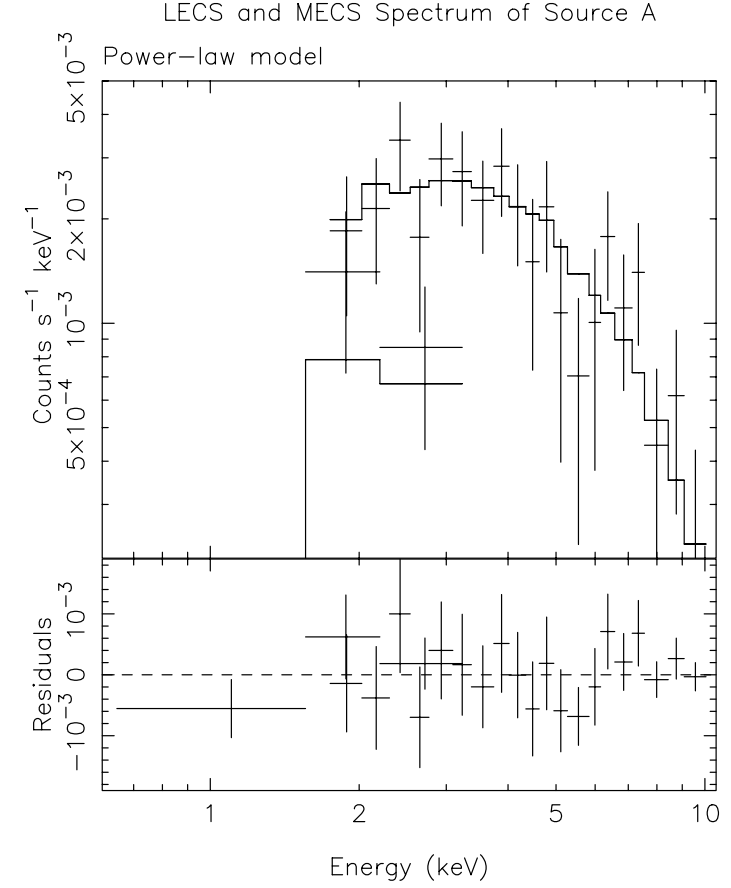

Fig. 4. BeppoSAX LECS and MECS spectrum of Source A. The best-fit power-law model is also shown together with the residuals in counts $\mathrm{s}^{-1} \mathrm{keV}^{-1}$

description of the spectrum. In agreement to the findings obtained with hardness ratios, the best-fit temperature of the MEKAL fit is very high and indicates that the power-law model may provide a more reasonable representation of the spectrum. Figure 4 shows the observed count spectrum together with the best-fit power-law model, and Table 2 summarizes the fit results. We have verified that the HPGSPC count-rate predicted by the best-fit models is a factor of 5 below the upper limit given in Sect. 2 .

For the Source C, we extracted the spectrum from a circular region of $5^{\prime}$ radius, which corresponds to the $90 \%$ encircled energy radius of the PSPC PSF at $50^{\prime}$ off-axis. The background was extracted from an annulus between $5^{\prime}$ and $8^{\prime}$. The spectrum was rebinned into 10 channels to oversample the energy resolution of the instrument and was fit with the same emission models as before using the appropriate response matrix and the ancillary file created with the task PCARF of FTOOLS 5.0. The background subtracted spectrum has 466 counts. The fit results are summarized in Table 2. Both thermal and non-thermal models are formally acceptable, but the MEKAL model systematically underestimates the flux below $0.8 \mathrm{keV}$, while the Bremsstrahlung model provide the best $\chi^{2}$ value.

\section{Discussion}

The X-rays emission we have detected in the direction of G318.2+0.1 is not well correlated with the radio shell: essentially, we have failed to reveal the thermal X-ray emission which should trace the interaction between the remnant and the interstellar medium. We have already noted that we see an enhancement at $14^{\mathrm{h}} 53^{\mathrm{m}} 40^{\mathrm{s}}-58^{\circ} 48^{\prime}$ which 
Table 2. Best-fit spectral parameters for the 2 strongest X-ray sources within G318.2+0.1. The flux is unabsorbed in the 2-10 keV energy range for BeppoSAX and 0.1-2.4 keV energy range for ROSAT in units of $10^{-12} \mathrm{erg} \mathrm{cm}^{-2} \mathrm{~s}^{-1}$. For the MEKAL fits the abundances were fixed at solar values. $\gamma$ is the power-law photon index and $k T$ is temperature. dof is the number of degrees of freedom

\begin{tabular}{|c|c|c|c|c|}
\hline Model & Parameter & $\begin{array}{c}N_{\mathrm{H}} \\
\left(\mathrm{cm}^{-2}\right)\end{array}$ & $\begin{array}{c}\text { Flux } 10^{-12} \\
\left(\operatorname{erg~cm} \mathrm{cm}^{-2} \mathrm{~s}^{-1}\right)\end{array}$ & $\chi^{2} /$ dof \\
\hline & \multicolumn{4}{|c|}{ Source A (LECS and MECS) } \\
\hline Power-law & $\gamma=1.8_{-0.7}^{+0.8}$ & $1.4_{-0.6}^{+3.5} 10^{22}$ & $1.2_{-0.5}^{+0.8}$ & $13 / 20$ \\
\hline MEKAL & $k T>2.6$ & $1.7_{-1.0}^{+2.6} 10^{22}$ & $1.2_{-0.2}^{+1.5}$ & $12 / 20$ \\
\hline BREMSS & $k T>4.7$ & $1.7_{-0.6}^{+1.0} 10^{22}$ & $1.2_{-0.2}^{+0.2}$ & $13 / 20$ \\
\hline \multirow[t]{2}{*}{ Black-body } & $k T=1.5_{-0.3}^{+0.3}$ & $<1.510^{22}$ & $0.9_{-0.2}^{+0.3}$ & $18 / 20$ \\
\hline & \multicolumn{4}{|c|}{ Source C (PSPC) } \\
\hline Power-law & $\gamma=2.1_{-0.5}^{+0.5}$ & $1.6_{-1.1}^{+1.5} 10^{20}$ & $2.0_{-0.3}^{+0.3}$ & $23 / 17$ \\
\hline MEKAL & $k T=2.6_{-0.6}^{+3.4}$ & $<1.510^{19}$ & $1.2_{-0.2}^{+0.3}$ & $26 / 17$ \\
\hline BREMSS & $k T=1.1_{-0.4}^{+1.0}$ & $1.0_{-0.4}^{+0.7} 10^{20}$ & $1.6_{-0.5}^{+0.9}$ & $19 / 17$ \\
\hline Black-body & $k T=0.18_{-0.03}^{+0.02}$ & $<1.510^{19}$ & $1.2_{-0.1}^{+0.0}$ & $27 / 17$ \\
\hline
\end{tabular}

is located almost on top of the radio filament, but further and deeper observations are needed to confirm this detection. On the other hand, we have detected three X-ray sources in the direction of the SNR G318.2+0.1, which are rather compact and loosely correlated with the position of the radio shell.

We have investigated whether the X-ray emission of Sources A and C could be due to stars within the uncertainty regions. We extracted lists of stars from the USNO-A2.0 catalog (Monet 1998) within $2^{\prime}$ from Sources $\mathrm{A}$ and $\mathrm{C}$, and considered the brightest star in each list. For Source A, there is an object with $R=13.9$ and $B=16.9$ which implies an M2 main sequence star. However, using the relation $N_{\mathrm{H}}=1.7910^{21} \times 3.1 \times E_{B-V}$ (Predehl \& Schmitt 1995) with the observed $N_{\mathrm{H}} \sim 1.510^{22} \mathrm{~cm}^{-2}$ (Table 2), we estimate a $E(B-V) \sim 2.5$, and the correction to $B-R$ is certainly as large. Therefore, the star has an unabsorbed $B-R \sim 0.5$, which corresponds to an F star. Since the unabsorbed $V$ magnitude should be around 14 and the unabsorbed $0.3-3.5 \mathrm{keV}$ flux is 0.8-2.3 $10^{-12} \mathrm{erg} \mathrm{cm}^{-2} \mathrm{~s}^{-1}$, we calculate that the ratio of X-ray to optical flux $\left(f_{\mathrm{X}} / f_{V}\right)$ is at least a factor of 10 higher than expected for a F star (see e.g., Maccacaro et al. 1988). Similarly, from the observed $E(B-V)$ of the star GSC 08693-00036 in the uncertainty region of Source C, we infer a K0 main sequence star at 90 pc. This is in agreement with the parallax measured by Hipparcos (ESA 1997), which yields a lower limit of 11 pc to the distance to GSC 08693-00036. The observed 0.3-3.5 keV flux is $1-310^{-12} \mathrm{erg} \mathrm{cm}^{-2} \mathrm{~s}^{-1}$, therefore, following Maccacaro et al. (1988), its $f_{\mathrm{X}} / f_{V}$ is consistent with a $\mathrm{K}$ star. The plasma temperature derived by the fit is in agreement with the temperature expected in a stellar corona. Thus, while Source C may be identified with emission from a foreground star, Source A appears unlikely to be so.

Source B is a very interesting object since it is probably the X-ray counterpart of the IRAS 14498-5856 UC H II region and, to our knowledge, no other similar cases have been yet reported in the literature. Walsh et al. (1997), using the methanol maser velocity, reported a kinematic distance to IRAS $14498-5856$ of 4.3 or $11.1 \mathrm{kpc}$ (depending on whether the source is on the near, or the far side, of the Galaxy). Using a thermal plasma emission model with a temperature of few $\times 10^{7} \mathrm{~K}$ (the model compatible with the hardness ratio analysis, Fig. 3), the MECS Source B has a flux of $7.2 \pm 1.410^{-13} \mathrm{erg} \mathrm{cm}^{-2} \mathrm{~s}^{-1}$, corresponding to $1.5 \pm 0.310^{33} \mathrm{erg} \mathrm{s}^{-1}$ at a distance of $4.3 \mathrm{kpc}$ and $\sim 10^{34} \mathrm{erg} \mathrm{s}^{-1}$ at a distance of $11.3 \mathrm{kpc}$. The former luminosity is high even for a young intermediate $\left(2-5 M_{\odot}\right)$ or high mass $\left(>5 M_{\odot}\right)$ star, but flares can increase luminosity. Ozawa et al. (1999) detected a quiescent X-ray emission of $10^{32} \mathrm{erg} \mathrm{s}^{-1}$ from SSV63, a $2-5 M_{\odot}$ object in the dark cloud L1630, and a flare of $10^{33} \mathrm{erg} \mathrm{s}^{-1}$. Hamaguchi et al. (2000) detected a flare from a more massive Herbig Be star of $510^{32} \mathrm{erg} \mathrm{s}^{-1}$. In all the cases, the measured temperature are between 1 and $10 \mathrm{keV}$, in agreement with the one inferred for Source B, and the flare timescale is larger than $10^{4} \mathrm{~s}$. Therefore, it may be the case that we have detected a flaring young massive star, even if the light-curve we have extracted in our $26 \mathrm{ksec}$ observation do not show particular evidence of variability. The large absorption we measure toward Source B is also in agreement with the explanation in terms of an embedded massive star, since the typical value for this kind of objects is above $10^{22} \mathrm{~cm}^{-2}$. If Source B is really the hard X-ray counterpart of the young massive star of the UC H II region, then we favor the lowest distance, because at $11 \mathrm{kpc}$ its luminosity would be higher than the one expected even in case of flare.

Alternatively, a population of low mass pre-main sequence stars can also produce hard X-rays. For instance, Hofner \& Churchwell (1997) propose this last explanation for the hard X-ray emission from the $\mathrm{H}$ II region $\mathrm{W} 3 \mathrm{ob}-$ served with ASCA with luminosity of $2.610^{33} \mathrm{erg} \mathrm{s}^{-1}$ in the $2-10 \mathrm{keV}$ energy range, similar to the luminosity of Source B. However, since the typical X-ray luminosity of low mass protostars are in the range $10^{30}$ to $10^{31} \mathrm{erg} \mathrm{s}^{-1}$, a large number of objects are required inside the BeppoSAX 
positional error circle of $2^{\prime}(2.5 \mathrm{pc}$ at $4.3 \mathrm{kpc})$ to account for the luminosity of our source. Unfortunatly, it is not clear if IRAS 14498-5856 is embedded in a larger star forming region. Further radio and X-ray observations are needed to study the environment of IRAS 14498-5856 and to better locate Source B, and therefore remove this ambiguity.

An independent hint about the nature of Source B comes from its IR flux. Using the flux densities at 60 and $100 \mu \mathrm{m}$ of 1133 and $2208 \mathrm{Jy}$ listed in the IRAS Point Source Catalog for this source and the relation $F_{\text {ir }}=1.310^{-11}\left(2.58 f_{60}+f_{100}\right)$ erg $\mathrm{cm}^{-2} \mathrm{~s}^{-1}$, adapted from Rice et al. (1988), we derive a far infrared luminosity of $1.410^{38} \mathrm{erg} \mathrm{s}^{-1}$, and an $F_{\text {ir }} / F_{\mathrm{x}}$ well above 100 , in agreement with the value expected from a star forming region (Vogler et al. 1997).

The association between SNRs and UC H II regions may be very common and, if established, supports a type II SN progenitor (Becker \& Helfand 1987). If $\mathrm{G} 318.2+0.1$ is associated with IRAS 14498-5856, then its diameter is $50 \mathrm{pc}$, and using the revised $\Sigma-\mathrm{D}$ relation given by Case \& Bhattacharya (1998) we estimate an expected radio surface brightness of 1-2 $10^{-21} \mathrm{~W} \mathrm{~m}^{-2} \mathrm{~Hz}^{-1} \mathrm{sr}^{-1}$ at $1 \mathrm{GHz}$. The observed surface brightness is $3.710^{-22} \mathrm{~W} \mathrm{~m}^{-2} \mathrm{~Hz}^{-1} \mathrm{sr}^{-1}$ and therefore this SNR is underluminous with respect to the $\Sigma-\mathrm{D}$ relation. This may imply an expansion into a low density medium, which in turn implies a strongly reduced contribution of the thermal X-ray emission due to the main shock, and a higher probability of observing non-thermal $\mathrm{X}$-ray emission due to accelerated electrons. This may explain why we do not see extended thermal emission along the shell. If Source A indeed has a hard non-thermal spectrum, it may be site of non-thermal electron acceleration. Similar hard point sources have been found inside IC 443 and are interpreted as evidence of slow shocks propagating into molecular clouds (Bocchino \& Bykov 2000). This scenario is very probable since Gaylard \& MacLeod (1993) and Green et al. (1997) have observed $1720 \mathrm{GHz}$ line emission inside this remnant at several locations, suggesting an $\mathrm{OH}$ maser due to shock excitation of neutral gas of molecular clouds. We strongly suggest further deep X-ray observations of this shell, in order to confirm the non-thermal nature of the emission, detect any diffuse emission at other locations in the shell, and to study the X-ray properties of the UC H II region coincident with Source B.

The interstellar absorption provides additional information about the distance. The absorption toward Source A is $0.8-4.910^{22} \mathrm{~cm}^{-2}$ (Table 2), which implies a distance of $2-14 / \overline{n_{0}} \mathrm{kpc}$, where $\overline{n_{0}}$ is the mean interstellar density in atoms $\mathrm{cm}^{-3}$. If the mean density is $\sim 1 \mathrm{~cm}^{-3}$, than the distance estimated in this way is in agreement with an association between Source A, Source B, the UC $\mathrm{H}$ II region and the SNR. On the other hand, the upper limit to the $N_{\mathrm{H}}$ is greater than the galactic absorption in this direction. As for Source $\mathrm{C}$ the low $N_{\mathrm{H}}$ implies a distance of $<1 \mathrm{kpc}$, which is consistent with the association with the K0 star located in the local cavity at $d<100 \mathrm{pc}$ studied by Sfeir et al. (1999).

Acknowledgements. BeppoSAX is a joint Italian-Dutch program. F. Bocchino acknowledges an ESA Research Fellowship. The MOST is operated by the University of Sydney with support from the Australian Research Council and the Science Foundation for Physics within the University of Sydney. This research has made use of data obtained through the High Energy Astrophysics Science Archive Research Center Online Service, provided by the NASA/Goddard Space Flight Center, and of data obtained through SIMBAD, provided by the Centre de Données astronomiques de Strasbourg.

\section{References}

Anders, E., \& Grevesse, N. 1989, Geocosmochim. Acta, 53, 197 Aschenbach, B., Brinkmann, W., Pfeffermann, E., Fuerst, E., \& Reich, W. 1991, A\&A, 246, L32

Becker, R. H., \& Helfand, D. J. 1987, ApJ, 316, 660

Bocchino, F., \& Bykov, A. M. 2000, A\&A, 362, L29

Boella, G., Butler, R. C., Perola, G. C., et al. 1997, A\&AS, 122, 299

Case, G. L., \& Bhattacharya, D. 1998, ApJ, 504, 761

ESA 1997, ESA SP-1200

Frontera, F., Costa, E., dal Fiume, D., et al. 1997, A\&AS, 122, 357

Gaylard, M. J., \& MacLeod, G. C. 1993, MNRAS, 262, 43

Giommi, P., Angelini, L., Jacobs, P., \& Tagliaferri, G. 1992, in ASP Conf. Ser. 25, Astron. Data Anal. Software Syst. I, 1, 100

Green, A. J., Frail, D. A., Goss, W. M., \& Otrupcek, R. 1997, AJ, 114, 2058

Hamaguchi, K., Terada, H., Bamba, A., \& Koyama, K. 2000, ApJ, 532, 1111

Hofner, P., \& Churchwell, E. 1997, ApJ, 486, L39

Maccacaro, T., Gioia, I. M., Wolter, A., Zamorani, G., \& Stocke, J. T. 1988, ApJ, 326, 680

Manzo, G., Giarrusso, S., Santangelo, A., et al. 1997, A\&AS, 122,341

Monet, D. E. A. 1998, in The PMM USNO-A2.0 Catalog. (1998), http://www.nofs.navy.mil

Morrison, R., \& McCammon, D. 1983, ApJ, 270, 119

Ozawa, H., Nagase, F., Ueda, Y., Dotani, T., \& Ishida, M. 1999, ApJ, 523, L81

Parmar, A. N., Martin, D. D. E., Bavdaz, M., et al. 1997, A\&AS, 122, 309

Pfeffermann, E., \& Aschenbach, B. 1996, in Roentgenstrahlung from the Universe, ed. H. U. Zimmermann, J. Trumper, \& H. Yorke, MPE Report 263, 267

Predehl, P., \& Schmitt, J. H. M. M. 1995, A\&A, 293, 889

Rice, W., Lonsdale, C. J., Soifer, B. T., et al. 1988, ApJS, 68, 91

Sfeir, D. M., Lallement, R., Crifo, F., \& Welsh, B. Y. 1999, A\&A, 346, 785

Voges, W., Aschenbach, B., Boller, T., et al. 1999, A\&A, 349, 389

Vogler, A., Pietsch, W., \& Bertoldi, F. 1997, A\&A, 318, 768

Walsh, A. J., Hyland, A. R., Robinson, G., \& Burton, M. G. 1997, MNRAS, 291, 261

White, N. E., Giommi, P., \& Angelini, L. 1994, IAU Circ., 6100,1

Whiteoak, J. B. Z., \& Green, A. J. 1996, A\&AS, 118, 329 